\title{
Methodology for kinematic cycle characterization of vehicles with fixed routes in urban areas
}

\author{
Felipe Jiménez*, Alfonso Román, José María López \\ University Institute for Automobile Research (INSIA), Technical University of Madrid (UPM), Campus Sur UPM, Carretera de Valencia km. 7, 28031 Madrid, Spain
}

\section{A R T I C L E I N F O}

\section{Keywords:}

Driving cycle

Traffic speed

Vehicles emissions

Urban buses

\begin{abstract}
A B S T R A C T
This paper analyses the driving cycles of a fleet of vehicles with predetermined urban itineraries. Most driving cycles developed for such type of vehicles do not properly address variability among itineraries. Here we develop a polygonal driving cycle that assesses each group of related routes, based on microscopic parameters. It measures the kinematic cycles of the routes traveled by the vehicle fleet, segments cycles into micro-cycles, and characterizes their properties, groups them into clusters with homogeneous kinematic characteristics within their specific micro-cycles, and constructs a standard cycle for each cluster. The process is used to study public bus operations in Madrid.
\end{abstract}

\section{Introduction}

This paper analyses the driving cycles of a vehicle fleet with predetermined urban itineraries. Over the years, driving cycles have been developed in many ways, often to meet data and experimental constraints, but sometimes to address particular issues. Most have limitations. At present, for example, the Economic Commission for Europe proposal is a linearized cycle, with its urban component developed in accordance with the traffic patterns of Paris in 1992, but this both needs augmenting to allow for extra-urban traffic and is limited by the assumption of a very smooth acceleration profile (Pelkmans and Debal, 2006). The US cycle FTP72 (LA-4) was designed to reproduce the conditions of traffic in the city of Los Angeles and is used in the US for vehicle emissions certification, which additionally requires passing trials involving aggressive driving conditions and circulation with air conditioning. The Highway Fuel Economy Cycle was developed to evaluate fuel consumption and emissions in nearly stationary highway traffic conditions. The list of cycles is long, and Table 1 offers a list of some that have been developed with an indication of their context.

The diversity of cycles poses problems in the selection of appropriate options in different contexts. Here we develop a methodology for the construction of polygonal driving cycles that reproduce the operation of vehicle fleets traveling fixed urban routes, while discriminating between differences in itineraries, seeking an approach that is easily replicated in a test bench.

\section{Methodology}

Following André (2004) we construct kinematic cycles that allow recording of driving conditions, analyses of the data and the development of the cycle, by applying the following sequence:

- Measurement of the kinematic cycles in the routes traveled by the vehicle fleet. 
Table 1

Some relevant driving cycles.

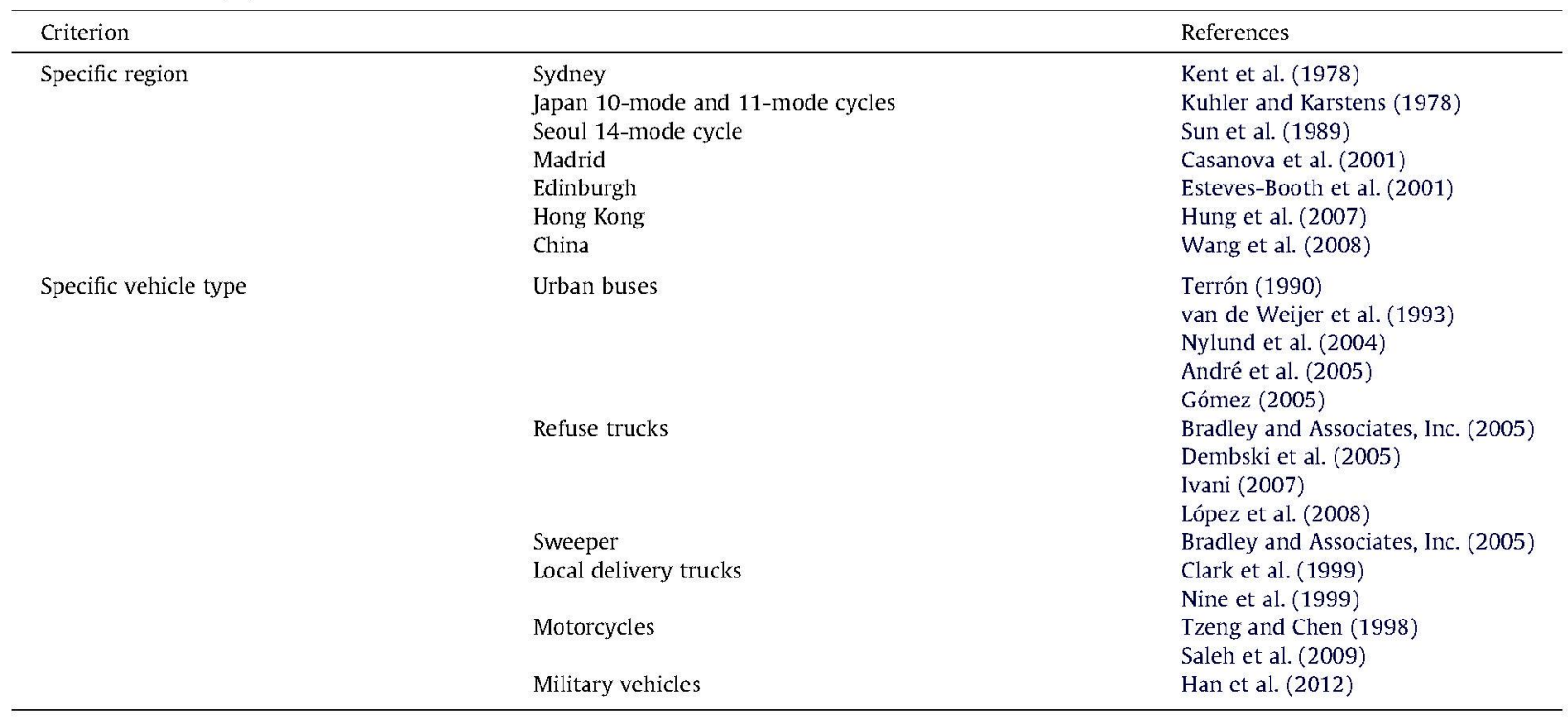

- Segmentation of the cycles in micro-cycles and characterization of their properties.

- Grouping of the routes into clusters with homogeneous kinematic characteristics within their specific micro-cycles.

- Construction of the corresponding cycle for each cluster of routes.

The methodology is applied to a bus routes in the city of Madrid, Spain.

Standard kinematic cycles are defined by the evolution of speed with respect to time, with the operating cycle divided into motion and stationary periods. In the data recordings, a micro-cycle corresponds to the evolution of the speed between two consecutive stops.

To obtain polygonal cycles, continuous recordings are categorized into micro-cycles composed of segments with constant acceleration, speed and deceleration to eliminate some oscillations from the original register. Movement micro-cycles with very low speeds and short durations are also discarded. While the only relevant information for stationary periods is their duration, the study of motion micro-cycles involves the utilization of the variables in Table 2 . In addition, Table 3 illustrates the classification of the polygonal micro-cycles into the diverse typologies found by linking sections in acceleration, constant speed and deceleration.

Route clustering is necessary to reflect that routes are unlikely to have similar kinematic characteristics, that a single polygonal cycle will not reproduce all itineraries, and that there are groups of routes with similar characteristics. André and Villanova (2004) group buses by operational peculiarities: route characteristics, travel time, irregularity of the journey,

Table 2

Variables analyzed in a micro-cycle.

\begin{tabular}{lll}
\hline Variable & Units & Symbol \\
\hline $\begin{array}{l}\text { Global } \\
\text { Duration }\end{array}$ & $\mathrm{s}$ & t_mean \\
$\begin{array}{l}\text { Average speed of the micro-cycle } \\
\text { Maximum speed in the micro-cycle }\end{array}$ & $\mathrm{km} / \mathrm{h}$ & Vmean \\
Acceleration & $\mathrm{km} / \mathrm{h}$ & Vmax \\
$\begin{array}{l}\text { Duration of the initial acceleration } \\
\text { Duration of the micro-cycle in acceleration }\end{array}$ & $\mathrm{s}$ & t_ac1 \\
$\begin{array}{l}\text { Initial acceleration in the micro-cycle } \\
\text { Constant speed }\end{array}$ & $\mathrm{s}$ & t_ttl_ac \\
Duration of the last section at constant speed & $\mathrm{m} / \mathrm{s}^{2}$ & ac1 \\
$\begin{array}{l}\text { Duration of the micro-cycle at constant speed } \\
\text { Velocity during last section at constant speed }\end{array}$ & $\mathrm{s}$ & $\mathrm{s}$ \\
$\begin{array}{l}\text { Deceleration } \\
\text { Duration of the final deceleration }\end{array}$ & $\mathrm{km} / \mathrm{h}$ & t_ttl_cru \\
$\begin{array}{l}\text { Duration of the micro-cycle in deceleration } \\
\text { Final deceleration in the micro-cycle }\end{array}$ & & $\mathrm{s}$ \\
\hline
\end{tabular}


Table 3

Classification of polygonal micro-cycles in accordance with its velocity.

\begin{tabular}{|c|c|c|}
\hline Classification & Morphology of the micro-cycle & Independent variables used to define the micro-cycle \\
\hline Type 1 & & ac1, decl, Vmax \\
\hline Type 2 & & ac1, decl, Vmax, tcrul \\
\hline Type 3 & & ac1, decl, Vmax, Vcru, t_crul, t_ttl_ac-t_ac1 \\
\hline Type 4 & & ac1, decl, Vmax, Vcru, t_crul, t_ttl_dec-t_decl \\
\hline Type 5A & & ac1, decl, Vmean, Vmax, t_ttl_ac-t_ac1, t_ttl_dec-t_decl, t_decl \\
\hline Type 5B & & ac1, decl, Vmean, Vmax, t_ttl_ac-t_ac1, t_ttl_dec-t_decl, t_ac1 \\
\hline Type 6A & & ac1, decl, Vmax, Vcru, t_ttl_ac-t_ac1, t_ttl_dec-t_decl, t_decl, t_crul \\
\hline Type 6B & & ac1, decl, Vmax, Vcru, t_ttl_ac-t_ac1, t_ttl_dec-t_decl, t_ac1, t_crul \\
\hline Type 7 & & ac1, decl, Vmean, Vmax, Vcru, t_ttl_ac-t_ac1, t_ttl_dec-t_decl, t_crul \\
\hline Type 8 & & ac1, decl, Vmean, Vmax, Vcru, t_ttl_ac-t_ac1, t_ttl_dec-t_decl, t_crul \\
\hline Type 9 & & ac1, decl, Vmax, t_ttl_ac-t_ac1, t_ttl_cru-t_crul, t_ac1, t_crul \\
\hline Type 10 & & ac1, decl, Vmax, Vcru, t_ttl_dec-t_decl, t_ttl_cru-t_crul, t_crul \\
\hline
\end{tabular}

Note: Typologies after 11 corresponds with the number of changes in slope that occur in the micro-cycle. 
etc., and here a clustering criterion reflecting the type of geographical area is added. The advantage of this is that line characterization is based on information exogenous to the bus' operations; the methodology evaluates macroscopic variables, and not microscopic, to denote the vehicle's operating conditions.

Data mining (Han et al., 2011) is used to group itineraries based on the micro-cycles' microscopic features. The degree of similarity between the routes is initially evaluated. For every route, the average value and a frequency histogram consisting of five intervals are calculated for each variable exposed in Table 2 . In addition, the proportions in which micro-cycle types appear are also analyzed. Thus, for each pair of routes, the null hypotheses of equal means and proportions in every histogram interval for each variable, and the equivalency of proportions for each micro-cycle typology are verified. From these comparisons, a symmetric matrix $M_{S}$ with the similarity indexes is obtained. Each element of this array has a value contained in $[0,1]$, corresponding to the ratio of contrasts that accept the null hypothesis compared to the contrasts performed (97). Additionally, a weighting factor is assigned to every variable to indicate the relevance of each contrast. The value of the element of matrix $M_{S}$ that compares route $i$ with $j$ is:

$$
M_{S}(i, j)=\frac{\sum_{k=1}^{N}\left(c_{k}^{m} \cdot h_{i j k}^{m}+\sum_{l=1}^{5} c_{k l}^{p} \cdot h_{i j k l}^{p}\right)+\sum_{q=1}^{M} c_{q}^{t} \cdot h_{i j q}^{t}}{\sum_{k=1}^{N}\left(c_{k}^{m}+\sum_{l=1}^{5} c_{k l}^{p}\right)+\sum_{q=1}^{M} c_{q}^{t}}
$$

where $N$ is the number of variables, $M$ is the quantity of micro-cycle types, $c^{m}$ is the weighting factor applied in the contrasts of average values, $c^{p}$ is the weighting factor used in the comparisons of variable proportion distributions, $c^{t}$ is the weighting factor utilized in the contrasts of micro-cycle type proportions, $h^{m}$ is a binary variable with a value of 1 when the null hypothesis in the comparison of averages is accepted and 0 when rejected, while similarly, $h^{p}$ equals one when the null hypothesis in the contrast of variable proportion distributions is fulfilled and zero if not and $h^{t}$ is one if the null hypothesis for the comparison of micro-cycle type proportions is verified and zero when refused.

The main objective for the grouping of routes is to generate clusters of lines that maximize a certain condition. With this purpose, two criteria are used:

- Maximum average value criterion. This condition measures the average value of all similarity indexes from every comparison that can be established between all itineraries belonging to each cluster. This grouping of routes maximizes:

$$
P=\frac{\sum_{i=1}^{C} \sum_{j=1}^{S_{i}} M_{S a b j}}{\sum_{i=1}^{C} S_{i}}
$$

where $C$ is the number of clusters, $S_{i}$ is the number comparisons for cluster $i$ and $M_{S a b}$ denotes, in a generic way, the terms of the similarity matrix for the prior comparisons.

- Maximum minimum value criterion. The prerequisite for this objective function is to maximize the minimum value of the similarity indexes obtained in the totality of the comparisons. The grouping of routes maximizes:

$$
M=\min \left(M_{\text {Sab }}\right)
$$

To monitor and measure the quality of the results, a number of indicators are also defined in terms of an average similarity index value indicator $(\mathrm{ps})$ and a minimum similarity index $(\mathrm{ms})$ of the lowest values in $M_{S}$ for all feasible comparisons between clustered routes.

The relative frequency distribution of the similarity indexes is also analyzed, and an additional requisite is established whereby, when reducing clusters, the initial groups must remain unaltered. Basically, once a pair of routes is assigned to a cluster, the association will not be withdrawn if $C$ decreases. This improves the level of consistency.

Three clustering methods are used and their performance compared.

- Combinatorial method. This consists of analyzing all combinations of clusters, assuming the number of routes and the preferred quantity of clusters are known. The main drawback is the large significant computational capacity required to generate the necessary combinations; the number of clusters to the power of the number of routes. Approximate methods may have to be adopted.

- Iterative method. Clusters are structured using a sequential process of assignations. Initially, the routes' ordering is arranged and this requires a criterion. Subsequently, taking into consideration each route in the pre-established order, if its similarity index with all other routes already belonging to a specific cluster exceeds a given tolerance value, the element is integrated within that group. Otherwise, comparison is made with the following grouping until the appropriate assembly is encountered. In the event that the route lacks an adequate similarity with any existing assemblies, a new cluster is created. Once the assignment process is completed, if the number of existing groups matches the predetermined quantity, the algorithm ends. Otherwise, the value of the tolerance is reduced to ease the cluster formation condition, thus starting a new iteration.

- Hierarchical clustering method. This consists of an iterative algorithm that performs a sequential grouping of routes into clusters; each cluster initially contains a single element. At every stage, all combinations of two clusters generated from the existing groups are calculated and the best is selected so that each step entails the union of two clusters into a new 
group. To select the most appropriate combination, the maximum $p s$ and $m s$ values are evaluated at each step. These requisites do not guarantee that the global optimum is reached, since previous groupings condition the successive arrangement of clusters.

The synthesis of polygonal driving cycles is based on a concatenation of micro-cycles intended to resemble the typology proportions and kinematic characteristics of those representing reality. Therefore, the most common micro-cycles are selected and each element is repeated with a frequency that is proportional to its existing relation with the remaining types, and which has been previously obtained from the actual measurements.

As a result of the high variability expected in the relative frequencies of the types of micro-cycles, the algorithm for cycle construction establishes two groups, with a slightly different treatment applied to each category:

- Class A: Micro-cycle types with relative frequencies exceeding above $10 \%$. The frequency of each configuration of independent variables in Table 3 is analyzed by defining five intervals for each. The most representative configurations are selected and each pattern is repeated to ensure that the relative frequency ratio amongst configurations conforms to the experimental recordings.

- Class $B$ : Micro-cycle types with low relative frequencies. Only the main configuration with the highest presence is utilized to represent the typology. Such micro-cycle is then replicated so that its frequency ratio with the remaining types is consistent.

The benchmark unitary frequency (the polygonal micro-cycle that is only repeated once during the cycle's construction) is the micro-cycle with the lowest occurrence ratio, when comparing the frequencies of each micro-cycle selected from class $A$ and with those included in class $B$, that is:

$$
\begin{array}{ll}
f_{u}=\min \left(f_{A i k}, f_{B j}\right) & j=1: I \\
& k=1: J \\
& =1: K_{i}
\end{array}
$$

where $I$ is the number of micro-cycle types in class $A, J$ its quantity for class B and $I+J$ the number of typologies considered in the study. $K_{i}$ is the number of configurations corresponding to micro-cycle type $i$ belonging to class $A, f_{A i k}$ represents the frequency of each configuration $k$ of the independent variables defining micro-cycle type $i$ belonging to class $\mathrm{A}$, and $f_{B j}$ the frequency of micro-cycle typology $j$ included in class B, fulfilling:

$$
\sum_{i=1}^{I} \sum_{k=1}^{K_{i}} f_{A i k}+\sum_{j=1}^{J} f_{B j}=1
$$

This algorithm selects the most frequent micro-cycles, while maintaining the existing proportions between typologies requiring compilation of:

- Frequency ratio among the configurations of micro-cycles belonging to the same type of class A: $f_{\text {Aik }} / f_{\text {Aip }}$.

- Frequency proportion between micro-cycles types of class A or B: $\left(f_{A i} / f_{A x}\right),\left(f_{B j} j f_{B y}\right)$.

- Frequency ratio amongst each micro-cycle type of class $A$ and class B: $f_{A i} / f_{B j}$.

Finally, the stationary segments are inserted between adjacent movement micro-cycles to obtain a frequency distribution for motionless time periods consistent with the data.

\section{Application}

The methodology is applied to 25 out of the 167 of the city bus routes in Madrid, excluding scheduled night and special services. They cover the main areas of the city and, according to the Municipal Bus Company of Madrid (EMT), are a good representation of the city's public bus operations.

The characterization of the kinematics among the routes is done using a purpose built, on-vehicle, data-logger device. The equipment operates autonomously, without driver involvement, recording analog and digital signals, and includes an internal accelerometer with $\mathrm{a} \pm 2 \mathrm{~g}$ measurement range on the longitudinal and transverse axes. The data registration is continuous, with an acquisition frequency of $1 \mathrm{~Hz}$ and records:

- Ignition, which activates or stops the data recording.

- Speed, obtained from the vehicle's dashboard, and calibrated in track tests performed for each bus by comparing its measurements with those provided by an L-EC Correvit non-contact speed sensor.

- Distance, also acquired from the information proceeding from the dashboard.

- Final stop display, which determines the beginning and end of the line's itinerary. 
Data were collected from a representative vehicle for each of the routes during a complete week; over $1000 \mathrm{~h}$ of data. As a starting point for the clustering algorithms, the similarity matrix $M_{S}$ is computed using a 0.01 significance level for hypothesis testing. The elements of the similarity matrix of dimension $25 \times 25$ vary between 0.978 and 0.235 . Although data are available for 25 routes, the combinatorial method becomes computationally infeasible for so many dimensions. Consequently, initial comparisons are conducted on six routes (L1-L6), and Table 4 illustrates comparisons involving the various cluster formation methods:

- C1: Combinatorial with maximum average value selection criterion.

- C2: Combinatorial with the maximum-minimum value preferred.

- I1: Iterative with initial arrangement of routes based on decreasing values of the sum of similarity indexes for each itinerary.

- 12: Iterative with a random initial sequence of routes.

- J1: Hierarchical clustering by implementing the maximum ps selection approach.

- J2: Hierarchical clustering with the maximum $m s$ value criterion.

The clusters to be accomplished vary from five to the lower limit of two, and the resulting arrangements are shown in Table 4. The results indicate that all methodologies support the same combination when more than three clusters are required. However, $\mathrm{C} 1$ provides a solution with the highest average index indicator, although it reduces the minimum index indicator to 0.637 due to the grouping of routes L1 and L5. Consequently, this solution provides a slightly higher average index than those obtained with the other methods, but originates a significant decrease in the minimum index, which for the remaining methods is 0.682 and 0.721 , respectively. Concerning the solution obtained using $C 2$ and 22 , although accounting for the highest minimum index, its average is the lowest. When the number of clusters is reduced to two, the values of the indicators denote that the preferable solution is obtained by grouping L1 and L5, with the remaining routes included within the other cluster formation. The solution provided by method 2 has the same minimum index, but its average index indicator is lower. The hierarchical J1 always maintains L1 within a single element cluster. The iterative method corroborates its dependence on the initial ordering of routes, with each of the criteria providing solutions when less than four clusters are required. The hierarchical method generates dissimilar results with three and two clusters because the group structure conditions the formation of successes. It is the only approach, however, that ensures consistency in the clustering process when the number of groups is reduced; the methodology does not rupture previous cluster formations obtained when there are more arrangements, which is preferred when adding new routes to existing structured clusters.

When the quantity of routes increases, the combinatorial method is not feasible. Consequently, in the analysis of 25 routes, only the iterative and hierarchical methods are contrasted. In this situation, the study examines the formation from 15 to 2 clusters. The values of the related quality indicators are seen in Figs. 1 and 2.

Table 4

Cluster formation and quality indicator values (six routes).

\begin{tabular}{|c|c|c|c|c|c|c|}
\hline Method & $\mathrm{C} 1$ & $\mathrm{C} 2$ & 11 & 12 & $\mathrm{~J} 1$ & $\mathrm{~J} 2$ \\
\hline \multicolumn{7}{|c|}{5 Clusters } \\
\hline$m s$ & 0.855 & 0.855 & 0.855 & 0.855 & 0.855 & 0.855 \\
\hline ps & 0.855 & 0.855 & 0.855 & 0.855 & 0.855 & 0.855 \\
\hline C. 1 & $\mathrm{~L} 1$ & $\mathrm{~L} 1$ & L1 & $\mathrm{L} 1$ & $\mathrm{~L} 1$ & L1 \\
\hline C. 2 & L2 L6 & L2 L6 & $12 \mathrm{~L} 6$ & L2 L6 & L2 L6 & L2 L6 \\
\hline C. 3 & $\mathrm{~L} 3$ & $\mathrm{~L} 3$ & $\mathrm{~L} 3$ & $\mathrm{~L} 3$ & $\mathrm{~L} 3$ & $\mathrm{~L} 3$ \\
\hline C. 4 & $\mathrm{~L} 4$ & $\mathrm{~L} 4$ & $\mathrm{~L} 4$ & $\mathrm{~L} 4$ & $\mathrm{~L} 4$ & $\mathrm{~L} 4$ \\
\hline C. 5 & $\mathrm{~L} 5$ & $\mathrm{~L} 5$ & $\mathrm{~L} 5$ & $\mathrm{~L} 5$ & $\mathrm{~L} 5$ & $\mathrm{~L} 5$ \\
\hline \multicolumn{7}{|c|}{4 Clusters } \\
\hline$m s$ & 0.844 & 0.844 & 0.844 & 0.844 & 0.844 & 0.844 \\
\hline ps & 0.847 & 0.847 & 0.847 & 0.847 & 0.847 & 0.847 \\
\hline C. 1 & $\mathrm{~L} 1$ & L1 & L1 & $\mathrm{L} 1$ & $\mathrm{~L} 1$ & L1 \\
\hline C. 2 & L2 L4 L6 & L2 L4 L6 & L2 L4 L6 & $\mathrm{L} 2 \mathrm{~L} 4 \mathrm{~L} 6$ & L2 L4 L6 & L2 L4 L6 \\
\hline C. 3 & $\mathrm{~L} 3$ & $\mathrm{~L} 3$ & $\mathrm{~L} 3$ & $\mathrm{~L} 3$ & $\mathrm{~L} 3$ & $\mathrm{~L} 3$ \\
\hline C. 4 & $\mathrm{~L} 5$ & $\mathrm{~L} 5$ & $\mathrm{~L} 5$ & $\mathrm{~L} 5$ & $\mathrm{~L} 5$ & $\mathrm{~L} 5$ \\
\hline \multicolumn{7}{|c|}{3 Clusters } \\
\hline$m s$ & 0.637 & 0.721 & 0.682 & 0.721 & 0.682 & 0.682 \\
\hline$p s$ & 0.795 & 0.767 & 0.788 & 0.767 & 0.788 & 0.788 \\
\hline C. 1 & L1 L5 & L1 L6 & L1 & L1 L6 & $\mathrm{L} 1$ & L1 \\
\hline C. 2 & $\mathrm{~L} 2 \mathrm{~L} 4 \mathrm{~L} 6$ & $\mathrm{~L} 2 \mathrm{~L} 3 \mathrm{~L} 4$ & L2 L3 L4 L6 & $\mathrm{L} 2 \mathrm{~L} 3 \mathrm{~L} 4$ & L2 L3 L4 L6 & L2 L3 L4 L6 \\
\hline C. 3 & $\mathrm{~L} 3$ & $\mathrm{~L} 5$ & $\mathrm{I} 5$ & $\mathrm{~L} 5$ & $\mathrm{~L} 5$ & $\mathrm{~L} 5$ \\
\hline \multicolumn{7}{|c|}{2 Clusters } \\
\hline$m s$ & 0.637 & 0.637 & 0.637 & 0.637 & 0.542 & 0.637 \\
\hline ps & 0.766 & 0.766 & 0.766 & 0.727 & 0.723 & 0.766 \\
\hline C. 1 & L1 L5 & L1 L5 & L1 L5 & L1 L5 L6 & $\mathrm{L} 1$ & L1 L5 \\
\hline C. 2 & L2 L3 L4 L6 & L2 L3 L4 L6 & $121314 \mathrm{~L} 6$ & $\mathrm{~L} 2 \mathrm{~L} 3 \mathrm{~L} 4$ & L2 L3 L4 L5 L6 & L2 L3 L4 L6 \\
\hline
\end{tabular}




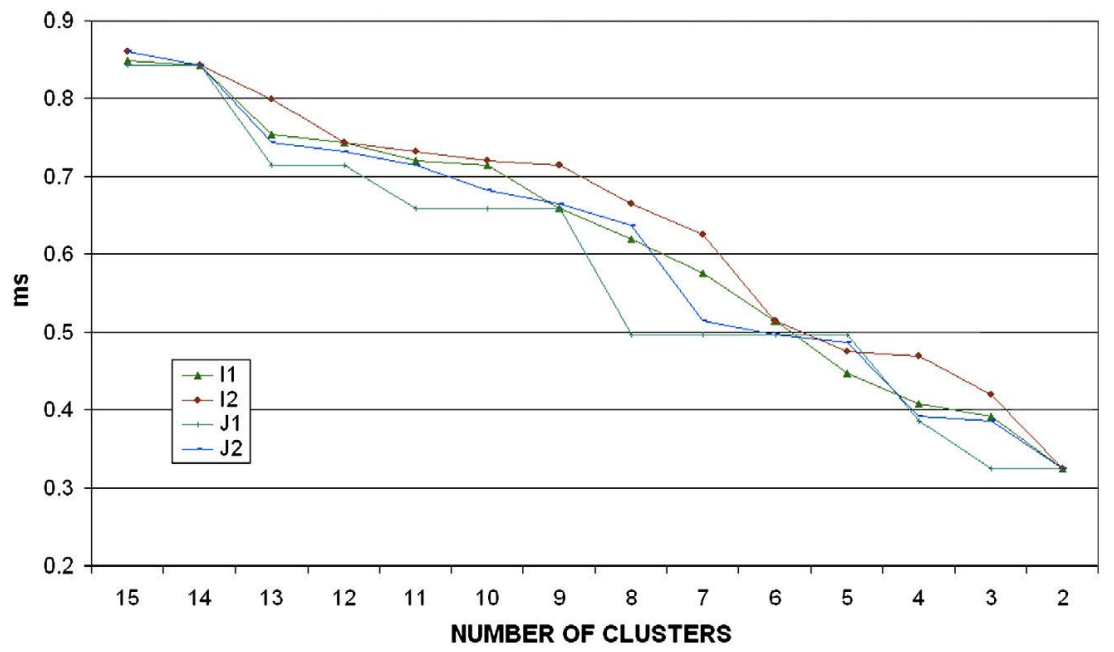

Fig. 1. Minimum index quality indicator $m s$ according to methodology (25 routes)

Iterative methods are inconvenient because they may mean initial routes are similar and tend to be clustered, thus limiting successive groupings. In conclusion, the application of the iterative method prevents achieving an adequate level of control over the quality of the results, since these depend on the initial ordering of the routes. The hierarchical method, due to its peculiar systematic, is the only methodology that provides consistent results throughout the complete sequence of clusters when their quantity is reduced. The version based on the optimization of the average index, however, selects arrangements without ensuring an adequate control over the minimum index's value. Consequently, for this application, this approach is not ideal, since it is undesirable to accept combinations with a low similarity index, even though the overall average may be high because of other comparisons. Based on previous considerations, the methodology that offers the best performance is the hierarchical method with the maximum-minimum selection criterion.

When the number of clusters is not predetermined, the analysis of Figs. 1 and 2 facilitates the decision as to which is the most convenient quantity. Usually, each reduction step in the number of clusters tends to decrease both quality indicators. The adequate amount of clusters is obtained when its succeeding negative slope decreases significantly. Such case is present in the $\mathrm{J} 2$ method when the $m s$ indicator varies from eight to seven clusters and from seven to six for $p s$ (the remaining abrupt changes occur for cluster quantities which are either too high or excessively low). Consequently, the selection of eight clusters provides a satisfactory value for both indicators.

Finally, the complete cycle is generated for each cluster of routes. With this intention, micro-cycle typologies between types 1 and 10 are considered. Likewise, relative frequencies of micro-cycles types 1 and 2 are verified to be well above $10 \%$ in all clusters, so these are classified as class $\mathrm{A}$, while the remaining types contain frequencies below or well-below this level, thus being categorized as class $\mathrm{B}$.

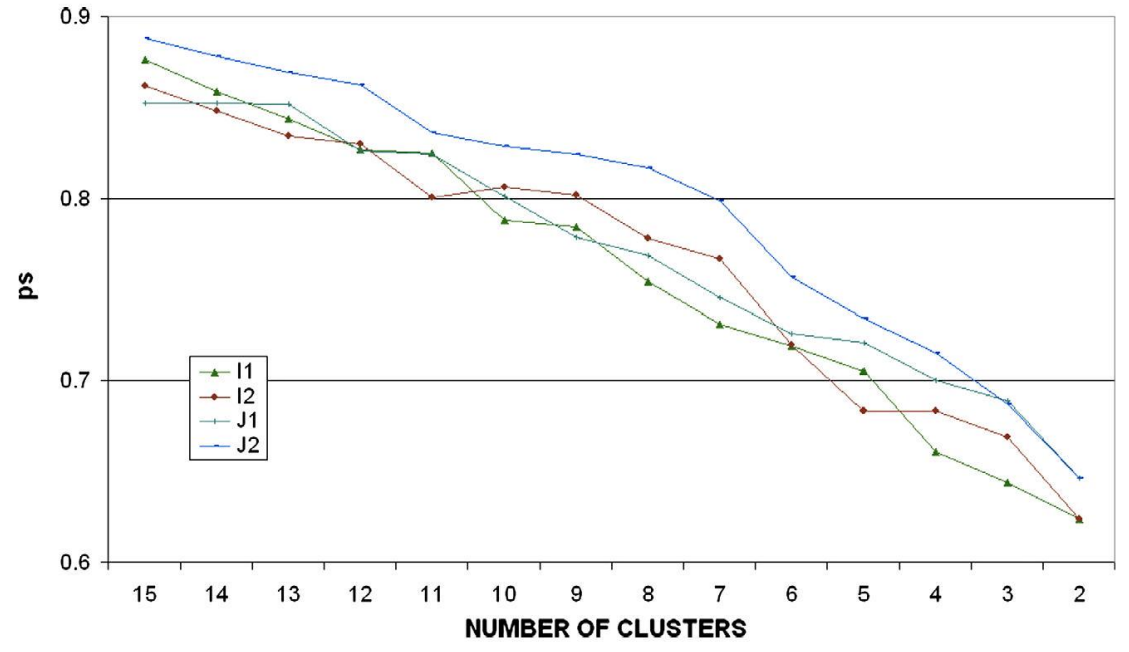

Fig. 2. Average index quality indicator ps according to the methodology (25 routes). 
Table 5

Characteristics of the complete generated cycles.

\begin{tabular}{|c|c|c|c|c|c|c|c|c|}
\hline Cluster & 1 & 2 & 3 & 4 & 5 & 6 & 7 & 8 \\
\hline \multicolumn{9}{|c|}{ Duration of the cycles } \\
\hline Movement & 1984.5 & 1867.4 & 2824.5 & 2969.6 & 2007.8 & 2615.8 & 2785.0 & 1527.1 \\
\hline Stationary & 1493.0 & 1600.0 & 2096.0 & 2348.0 & 1267.0 & 1680.0 & 1540.0 & 714.0 \\
\hline Total & 3477.5 & 3467.4 & 4920.5 & 5317.6 & 3274.8 & 4295.8 & 4325.0 & 2241.1 \\
\hline \multicolumn{9}{|c|}{ Number of micro-cycles of each typology } \\
\hline $1^{\mathrm{a}}$ & $30(5)$ & $36(3)$ & $53(5)$ & $65(6)$ & $27(3)$ & $42(6)$ & $39(7)$ & $11(3)$ \\
\hline $2^{\mathrm{a}}$ & $14(12)$ & $14(10)$ & $15(11)$ & $15(13)$ & $15(12)$ & $15(12)$ & $15(9)$ & $15(12)$ \\
\hline 3 & 2 & 2 & 2 & 3 & 3 & 4 & 3 & 1 \\
\hline 4 & 2 & 2 & 2 & 2 & 2 & 3 & 3 & 1 \\
\hline $5 \mathrm{~A}$ & 5 & 4 & 7 & 7 & 5 & 7 & 7 & 2 \\
\hline $5 \mathrm{~B}$ & 2 & 1 & 2 & 3 & 2 & 2 & 0 & 1 \\
\hline $6 \mathrm{~A}$ & 0 & 0 & 0 & 0 & 0 & 0 & 2 & 0 \\
\hline $6 \mathrm{~B}$ & 0 & 0 & 0 & 0 & 0 & 0 & 0 & 0 \\
\hline 7 & 2 & 1 & 2 & 2 & 1 & 2 & 1 & 2 \\
\hline 8 & 2 & 1 & 1 & 1 & 1 & 2 & 1 & 1 \\
\hline 9 & 0 & 1 & 1 & 0 & 1 & 1 & 1 & 1 \\
\hline 10 & 1 & 1 & 1 & 0 & 1 & 1 & 2 & 1 \\
\hline \multicolumn{9}{|c|}{$\%$ of disregarded micro-cycles type 11 or above } \\
\hline & $7.8 \%$ & $6.6 \%$ & $6.0 \%$ & $4.2 \%$ & $11.2 \%$ & $18.0 \%$ & $37.2 \%$ & $19.2 \%$ \\
\hline \multicolumn{9}{|c|}{ Similarity index values obtained from the comparison of the standard cycle with the registered information } \\
\hline & 0.844 & 0.676 & 0.804 & 0.810 & 0.698 & 0.637 & 0.508 & 0.709 \\
\hline
\end{tabular}

a The number of micro-cycles is indicated first; the parenthesis designates the quantity of configurations involved.

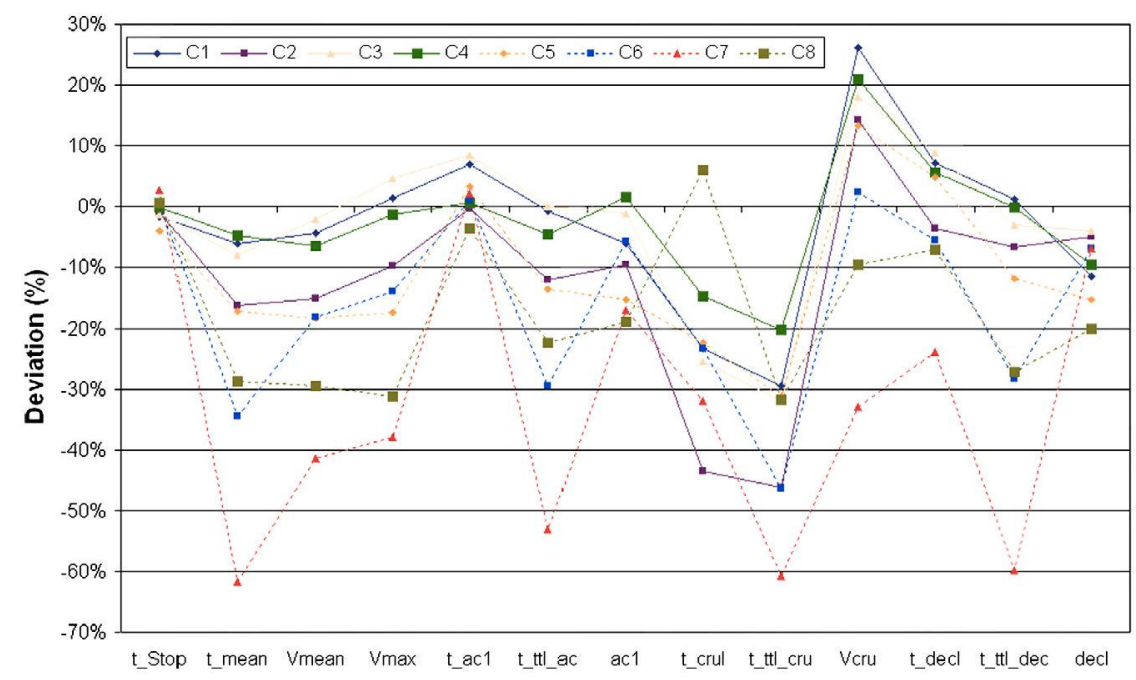

Fig. 3. Deviations in the most relevant variables describing the standard cycles and the experimental data.

The characteristic micro-cycles are then constructed from previous selections of configurations by computing the dependent variables. Next, micro-cycle concatenation is applied implementing the repetitions needed to maintain the actual relative frequency proportions present within the typologies. To obtain comparable results, a fairly fixed number of type 2 micro-cycles is selected, 14 or 15 , depending on differences due to rounding, with the lowest frequency being the reference for calculating the adequate proportions for the remainder.

The main characteristics of the complete generated cycles are shown in Table 5. As may be observed, a wide diversity of results is obtained for the various clusters. Hence, the cycle time varies between $0.62 \mathrm{~h}$ and $1.48 \mathrm{~h}$, values which are in the order of magnitude, or lengthier, of other standard cycles presently being utilized. However, for all clusters, there is greater homogeneity in the proportion of cycle types 1 and 2 , with its incidence ranging between $72 \%$ and $82 \%$.

Validation of the results involves comparing the values of the characteristic variables defining the micro-cycles of the standard generated cycles with the equivalent recorded data for each cluster. The similarity index following Eq. (1), which results from the comparison of the constructed cycle and all real measurements, is also calculated for every cluster. Fig. 3 illustrates the existing deviations for the most relevant variables. These results indicate that for some clusters (especially 
groupings 1,3 and 4 ), its simulated cycle has a satisfactory resemblance with the measured data, presenting differences below $10 \%$ in all or almost all variables, with the most significant deviations existing among the variables relative to sections traveled at constant velocity. This occurs mainly in those routes in which the simplest cycles have a higher presence. Clusters $5-8$, however, contain significant frequencies of type 11 or above micro-cycles that have not been considered in the construction of the standard cycle, thus inducing larger divergences to the results of standard cycle from actual data. This is relevant in the case of cluster 7, for which these cycle types constitute over $37 \%$. Such clusters resemble routes of high average speed and few stops because a significant component of their itinerary involves non-urban transit. This indicates that the methodology's use is limited to urban areas.

\section{Conclusions}

We develop a methodology for characterization of standard driving cycles in a fleet of vehicles that traverse fixed routes in an urban region. A single cycle is not used to represent all routes, because it is likely that significant differences exist amongst them. To handle this we use a process that groups routes into clusters, using available microscopic variables. A series of algorithms and grouping criteria are evaluated. As a result, the hierarchical method with selective criterion that maximizes the least unfavorable combination of route groupings is preferred, because it is the only methodology providing consistent arrangements throughout the complete sequence of cluster formations, while also capable of providing acceptable values for the lowest similarity index.

\section{Acknowledgments}

This work has been partly funded by the Spanish Ministry of Environment and the EMT. The authors are also grateful for the support of EMT employees during the installation of recording equipment in the buses.

\section{References}

Andrê, M., 2004. Real-world Driving Cycles for Measuring Cars Pollutant Emissions - Part A: The ARTEMIS European Driving Cycles. INRETS-LTE 0411. Institute National de Recherche sur les Transports et leur Sécurité (INRETS), Bron.

André, M., Villanova, A., 2004. Characterization of an urban bus network for environment purposes. Science of the Total Environment 334-335, 85-99.

André, M., Garrot, B., Roynard, Y., Vidon, R., Tassel, P., Perret, P., 2005. Operating conditions of buses in use in the Ile-de-France region of France for the evaluation of pollutant emissions. Atmospheric Environment 39, 2411-2420.

Bradley and Associates, Inc., 2005. A Demonstration of Diesel Particulate Filter Emission Control Technologies on Refuse Collection Trucks and Deployment of Natural Gas Powered Street Sweepers. Cummins, Inc., Columbus.

Casanova, J., Valdés, M., Aríztegui, J., 2001. The influence of car fleet composition on urban pollutant emissions. Application to the city of Madrid. In: Proceedings of the 10th International Symposium Transport Air Pollution, Boulder.

Clark, N.N., Daley, J.J., Nine, R.D., Atkinson, C.M., 1999. Application of the New City-suburban Heavy Vehicle Route (CSHVR) to Truck Emissions Characterization. SAE Technical Paper 1999-01-1467, Warrendale.

Dembski, N., Rizzoni, G., Soliman, A., Fravert, J., Nelly, K., 2005. Development of Refuse Vehicle Driving and Duty Cycles. SAE Technical Paper 2005-01-1165, Warrendale.

Esteves-Booth, A., Muneer, T., Kirby, H., Kubie, J., Hunter, J., 2001. The measurement of vehicular driving cycle within the city of Edinburgh. Transportation Research Part D 6, 209-220.

Gómez, F., 2005. Contribución a la optimización energética de vehículos propulsados por sistemas híbridos. Technical University of Madrid, PhD Thesis (in Spanish).

Han, J., Kamber, M., Pei, J., 2011. Data Mining: Concepts and Techniques, third ed. Morgan Kaufmann Publishers, Burlington.

Han, D.S., Choi, N.W., Cho, S.L., Yang, J.S., Kim, K.S., Yoo, W.S., Jeon, C.H., 2012. Characterization of driving patterns and development of a driving cycle in a military area. Transportation Research Part D 17, 519-524.

Hung, W.T., Tong, H.Y., Lee, C.P., Ha, K., Pao, L.Y., 2007. Development of a practical driving cycle construction methodology: a case study in Hong Kong. Transportation Research Part D 12, 115-128.

Ivani, Z., 2007. Data Collection and Development of New York City Refuse Truck Duty Cycle. SAE Technical Paper 2007-01-4118, Warrandale.

Kent, J.H., Allen, G.H., Rule, G., 1978. A driving cycle for Sydney. Transportation Research 12, 147-152.

Kuhler, M., Karstens, D., 1978. Improved Driving Cycle for Testing Automotive Exhaust Emissions. SAE Technical Paper 780650, Warrendale.

López, J.M., Jiménez, F., Aparicio, F., Flores, N., 2008. Comparison of exhaust emissions of vehicles for garbage collection and tankers for streets cleaning using diesel and biodiesel. In: FISITA Congress, September 15-19, 2008, Munich.

Nine, R.D., Clark, N.N., Delay, J.J., Atkinson, C.M., 1999. Development of a heavy duty chassis dynamometer driving route. Proceedings of the Institution of Mechanical Engineers, D 213, 561-574.

Nylund, N.-O., Erkkilä, K., Lappi, M., Ikonen, M., 2004. Transit Bus Emission Study: Comparison of Emissions from Diesel and Natural Gas Buses. Research Project PRO3/P5150/04. VTT Technical Research Centre of Finland, Espoo.

Pelkmans, L., Debal, P., 2006. Comparison of on-road emissions with emissions measured on chassis dynamometer test cycles. Transportation Research Part D 11, 233-241.

Saleh, W., Kumar, R., Kirby, H., Kumar, P., 2009. Real world driving cycle for motorcycles in Edinburgh. Transportation Research Part D 14, 326-333.

Sun, P., Chul-Hong, K., Chan-Jo, K., 1989. Development of the Urban Driving Cycle in Seoul. SAE Technical Paper 891354, Warrendale.

Terrón, J.A., 1990. Modelización y optimización de la circulación de autobuses en circuitos de regulación semafórica. Technical University of Madrid, PhD Thesis (in Spanish).

Tzeng, G.-H., Chen, J.-J., 1998. Developing a Taipei motorcycle driving cycle for emissions and fuel economy. Transportation Research Part D: Transport and Environment 3, 19-27.

van de Weijer, C.J.T., van der Graff, R., Hendriksen, P., Verbeek, R.P., 1993. Urban bus driving cycle. In: 4th EAEC International Conference on Vehicle Safety and Traffic Systems Technology, Strasbourg.

Wang, Q., Huo, H., He, K., Yao, Z., Zhang, Q., 2008. Characterization of vehicle driving patterns and development of driving cycles in Chinese cities. Transportation Research Part D: Transport and Environment 13, 289-297. 